\title{
Size and shape effects on two-phase flow patterns in microchannel forced convection boiling
}

\author{
Man Lee ${ }^{1}$, Yiu Yan Wong ${ }^{1}$, Man Wong ${ }^{2}$ and Yitshak Zohar ${ }^{1}$ \\ ${ }^{1}$ Department of Mechanical Engineering, Hong Kong University of Science and Technology, \\ Clear Water Bay, Kowloon, HKSAR, People's Republic of China \\ ${ }^{2}$ Department of Electrical and Electronic Engineering, Hong Kong University of Science and \\ Technology, Clear Water Bay, Kowloon, HKSAR, People's Republic of China
}

Received 20 August 2002, in final form 20 November 2002

Published 12 December 2002

Online at stacks.iop.org/JMM/13/155

\begin{abstract}
An integrated microchannel heat sink consisting of shallow, nearly rectangular microchannels has been fabricated using standard micromachining techniques to highlight the effects of the micrometer sized channel shape on the evolving flow patterns and, consequently, on the thermal performance of the microsystem. An integrated heater serves as a local heat source, while an array of micro thermistors is used for temperature distribution measurements. The working fluid, DI water, is pressurized through the microchannels for forced convection heat transfer studies. Boiling curves for different flow rates have been recorded and analyzed based on the visualized flow patterns. Local nucleation, including bubble formation and bubble dynamics, is documented and found to be negligible. Although detected, in contrast with triangular microchannels, annular flow is observed to be unstable. Instead, the dominant flow pattern is an unsteady transition region connecting an upstream vapor zone to a downstream liquid zone with an average location depending on the input power. A physical mechanism based on the force balance across the vapor-liquid interface, and the development of a restoring force, is proposed to explain the flow visualization results.
\end{abstract}

M This article features online multimedia enhancements

\section{Introduction}

Two-phase convective flow has numerous promising applications such as cooling of electronic components [1]. The principal advantage of two-phase flow lies in the utilization of latent heat absorbed by the working fluid due to phase change from liquid to vapor without increasing the temperature. Furthermore, for unsteady thermal performance, the response time of either heating-up or cooling-down process under forced convection is significantly shorter than under free convection heat transfer [2].

Convective heat transfer in macrosystems has been studied extensively. However, research on the effects of the channel size and shape on two-phase flow patterns in microchannels is still very limited, especially in systems with a length scale of the order of $10 \mu \mathrm{m}$ or less. Understanding the mechanisms and fundamental differences involved with heat transfer in two-phase flow in microgeometries has become increasingly important [1]. In macrosystems, gravity and pressure gradient are the dominant forces while surface tension is negligible. On the other hand, gravity is negligible but surface tension becomes a dominant force in microsystems. Consequently, two-phase flow is an ideal example to illustrate the physics of scaling in microsystems.

The thermal performance of a microsystem depends on the evolving flow pattern in microchannels [3]. Peng and Wang [4] experimentally investigated the phase change in microchannels with a rectangular cross-section of $600 \times$ $700 \mu \mathrm{m}^{2}$, and reported that the boiling regime was fully developed nucleate boiling and no partial or local nucleate 


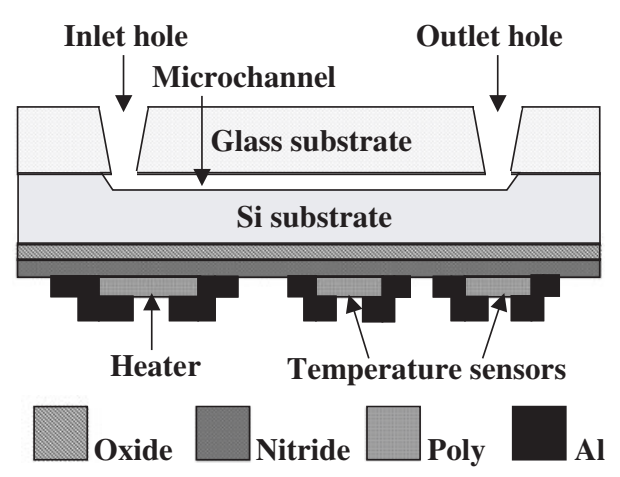

Figure 1. A schematic cross-section of the microchannel heat sink.

boiling was detected. In contrast, Jiang et al [5] recorded a periodic sequence of bubble formation, growth and departure from a few nucleation sites inside triangular microchannels with a hydraulic diameter as small as $26 \mu \mathrm{m}$. Furthermore, no plateau was observed in the boiling curves due to the stable annular flow, which developed at a relatively low input power. Peles et al [6] conducted an experiment and analysis of forced convection boiling in microchannel with hydraulic diameter of $157 \mu \mathrm{m}$, in which single bubbles as well as vaporliquid interface within the channels were observed. Hetsroni et al [3] experimentally demonstrated that flow boiling in microchannels, about $130 \mu \mathrm{m}$ in hydraulic diameter, resulted in a significant enhancement of the heat transfer. They reported the development of two-phase flow patterns resulting from hydrodynamic instabilities once phase change took place. Zhang et al [7] concluded that the dimensions of a rectangular microchannel were critical for the evolving flow pattern and the device thermal response. In a subsequent work, Zhang et al [8] attempted to enhance the heat transfer rate by creating small cavities in the channel sidewalls. In this work, a micro heat sink with nearly rectangular microchannels is designed and fabricated in order to investigate the size and shape effects on the development of two-phase flow patterns and the resulting temperature distributions.

\section{Device design and fabrication}

A micro heat sink, with high aspect ratio and nearly rectangular microchannels, was designed to study the size and shape effect on two-phase flow patterns developed during convective boiling. Standard micromachining techniques were utilized in the fabrication of the integrated microsystem. A schematic cross-section of the microdevice is shown in figure 1. The micro heat sink, fabricated using a p-type silicon wafer, consisted of ten microchannels, figure 2( $a$ ), an integrated local heater and a temperature microsensor array, figure $2(b)$. The integrated heater at one edge of the device functioned as the heat source. A $10 \times 10$ array of temperature microsensors was fabricated for detailed temperature distribution measurements. The heater and temperature sensors were formed using a selectively doped $0.4 \mu \mathrm{m}$ thick polysilicon film. A glass wafer, with inlet/outlet holes, was anodically bonded to the silicon substrate to cap the microchannels formed by Si etch in TMAH. A SEM picture of the nearly rectangular microchannel cross-section is shown in figure 3, where the depth is $14 \mu \mathrm{m}$ and the average width is $120 \mu \mathrm{m}$ (hydraulic diameter of $24 \mu \mathrm{m})$. The resulting microchannels with a transparent cover, about $19 \mathrm{~mm}$ in length, allowed video recording of the evolving flow patterns during the operation. The detailed fabrication process has been described elsewhere [9]. The overall area of a single die was about $12 \times 23 \mathrm{~mm}^{2}$. Each die was packaged and wire bonded to facilitate connections to the external fluid handling system as well as the electronic equipment for $\mathrm{I} / \mathrm{O}$ signals.

\section{Experimental setup and calibration}

The experimental setup is schematically illustrated in figure 4 . DI water contained in a pressurized tank was driven by adjustable high-pressure gas source without a degassing process. The DI water was forced through a $1 \mu \mathrm{m}$ filter to prevent particles from blocking the microchannels. After passing through the microdevice, the fluid exiting the micro heat sink was cooled down and condensed by immersing the outlet tube in cold water. The condensed water was then collected in a graduated cylinder to measure the volume flow rate. The micro heater was connected to a power source with adjustable voltage to provide heat to the device. The temperature sensor output signals were recorded using a computerized data acquisition system. Simultaneously, the flow patterns developed in the microchannels were recorded by a video-recording system, which included a CCD camera mounted on a microscope, a monitor and a VCR.

The temperature microsensors were calibrated prior to the experiments. The device was placed in an oven, and the resistance change of each sensor was recorded as a function of the controlled temperature change. The resulting calibration curves of the temperature microsensors are depicted in figure 5, where $T_{0}$ and $R_{0}$ are the room temperature and the corresponding resistance, respectively. The expected non-linear response with negative temperature coefficient of resistance (TCR) due to the phosphorus doping is evident, and the sensor performance is very uniform. The accuracy of the temperature measurements was within $\pm 0.5^{\circ} \mathrm{C}$.

\section{Temperature distribution and boiling curves}

The micro heat sink surface temperature was measured as a function of the input power under forced convection keeping a constant inlet pressure (controlled) and a constant outlet pressure (atmospheric). Two inlet pressures of 45 and $90 \mathrm{kPa}$ were selected corresponding to a constant low flow rate, $Q_{\mathrm{L}}=$ $0.0315 \mathrm{~mL} \mathrm{~min}^{-1}$, and high flow rate, $Q_{\mathrm{H}}=0.063 \mathrm{~mL} \mathrm{~min}^{-1}$, at room temperature. Since the spanwise distribution was found to be uniform [10], only the temperature distribution measurements $T(x)$ along the device centerline for the low and high flow rates are summarized in figures $6(a)$ and $(b)$, respectively, as a function of the input power level $q$. The missing data set in figure $6(b)$ is due to the failure of the temperature sensor located at $x=14 \mathrm{~mm}$. The temperature of the microdevice increases with input power. When the input power is low, the temperature distribution is almost 


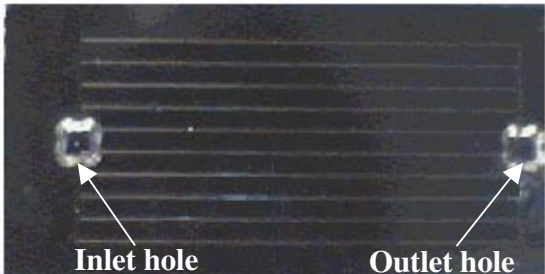

(a)

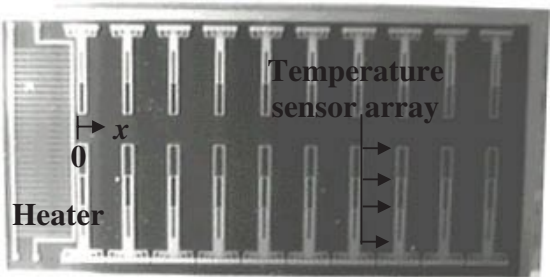

(b)

Figure 2. Pictures of a fabricated micro heat sink: (a) glass side, showing the microchannels with the inlet/outlet holes, and $(b)$ silicon side, showing the heater, temperature sensors and metal lines (die size: $12 \times 23 \mathrm{~mm}^{2}$ ).

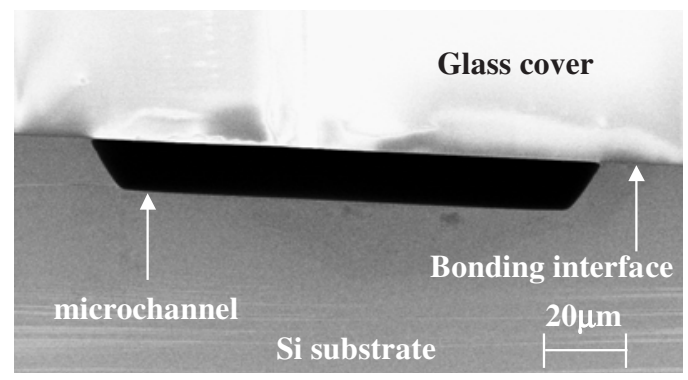

Figure 3. A SEM micrograph showing a cross-section of the nearly rectangular microchannel, $14 \mu \mathrm{m}$ in depth and $120 \mu \mathrm{m}$ in average width.

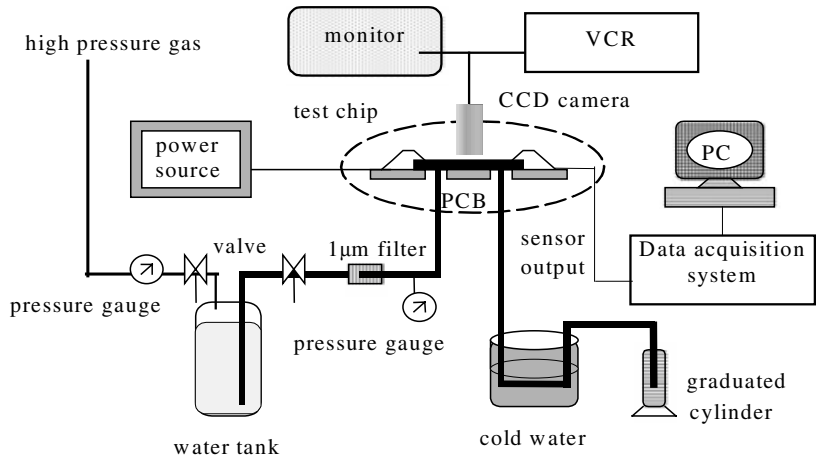

Figure 4. A schematic illustration of the experimental setup.

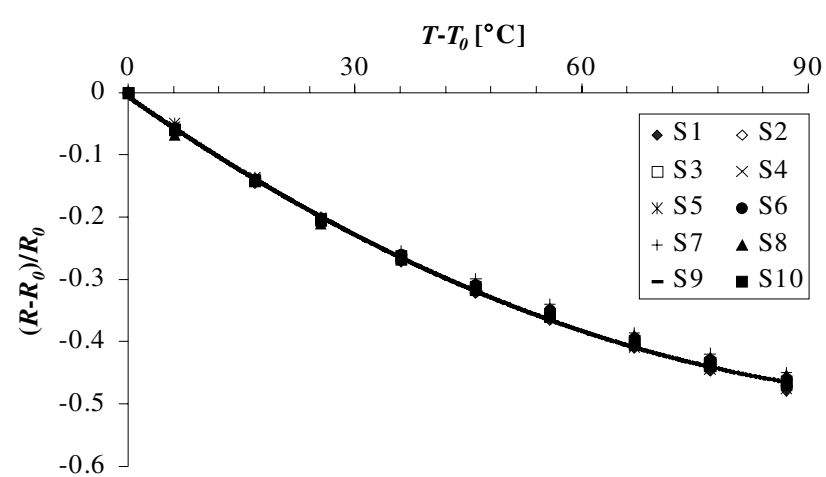

Figure 5. Calibration curves of the resistance change of the temperature sensors as a function of the temperature change.

uniform. As the input power is increased, the magnitude of the temperature gradient along the device also increases as demonstrated in figure $6(c)$.

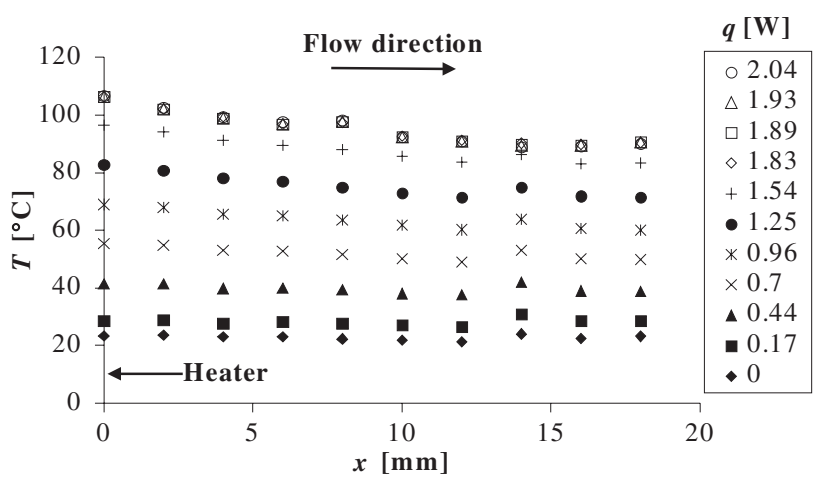

(a)

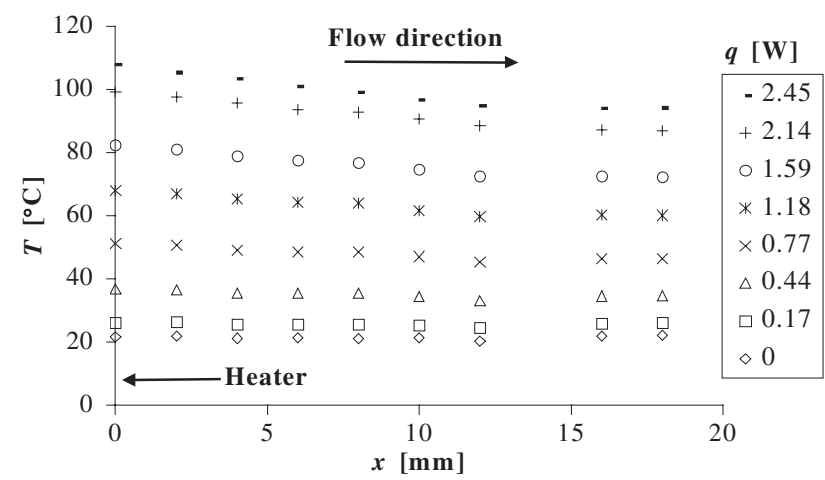

(b)

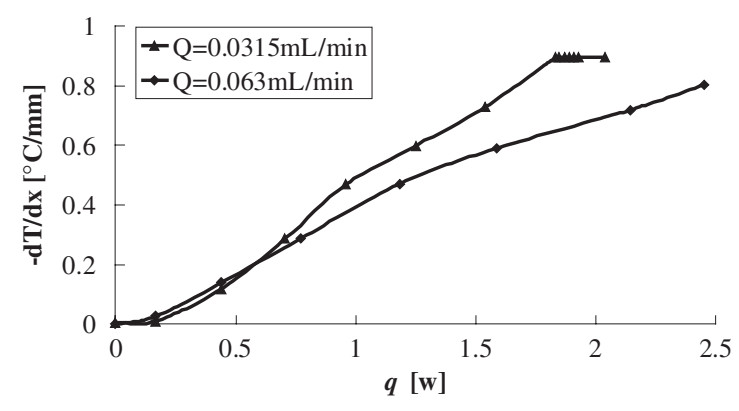

(c)

Figure 6. Temperature distribution along the device centerline, $T(x)$, at various levels of input power, $q$, for $(a)$ low water flow rate,

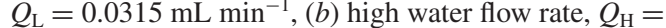

$0.063 \mathrm{~mL} \mathrm{~min}^{-1}$ and $(c)$ the corresponding temperature gradients.

The surface temperature is plotted as a function of the power dissipation, at a few selected locations along the device centerline, to construct the boiling curves shown in figure 7. 


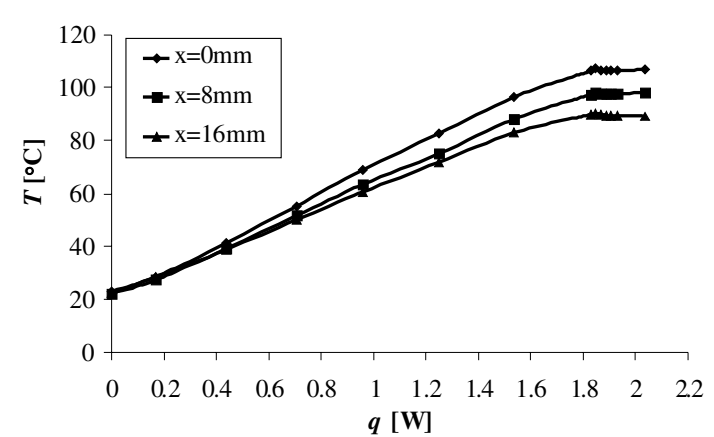

(a)

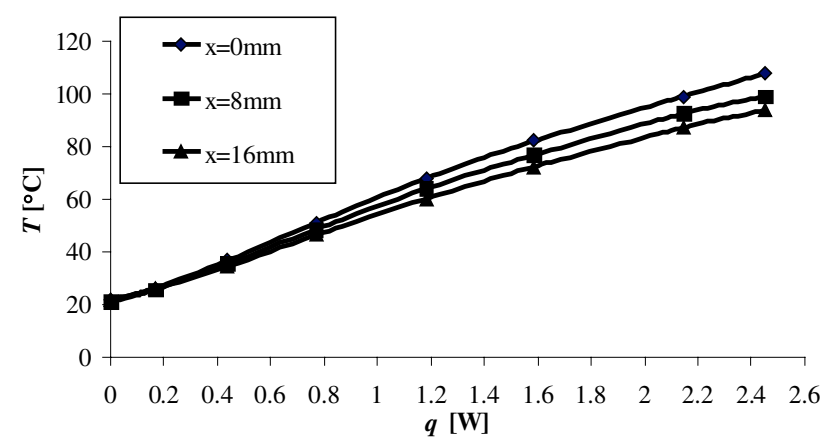

(b)

Figure 7. Boiling curves of the device surface temperature as a function of the input power under forced convection with flow rate of (a) $Q_{\mathrm{L}}=0.0315 \mathrm{~mL} \mathrm{~min}^{-1}$ and $(b) Q_{\mathrm{H}}=0.063 \mathrm{~mL} \mathrm{~min}^{-1}$.

Since the heater was located at one edge of the heat sink resulting in a non-uniform temperature distribution, the boiling curves do not collapse together. Nevertheless, all the curves exhibit similar trend independent of the downstream location. Initially, the temperature increases monotonically with the input power. However, at a power input close to the critical heat flux $(\mathrm{CHF})$ level, $q_{\mathrm{CHF}}$, the boiling curve characteristics depend on the water flow rate. For the low flow rate, figure 7(a), the curves do exhibit the boiling-plateau feature, $\partial T / \partial q \cong 0$, associated with latent heat of liquid-to-vapor phase change. At the low input power range, up to a level of about $1.85 \mathrm{~W}$, the heat is removed from the system through both forced convection by the working fluid and natural convection from the device surface. Consequently, the local temperature increases almost linearly with the input power. Further power increase leads to a phase change of the water from liquid to vapor, as the vapor void fraction increases with input power. Thus, the local temperature does not increase with increasing power, which is converted to latent heat of phase change. The onset of critical heat flux condition develops at a power level higher than $2.05 \mathrm{~W}$. However, for the high flow rate, figure $7(b)$, no plateau is observed in the boiling curves similar to previous reports $[5,10]$. The surface temperature monotonically increases with increasing input power until the onset of critical heat flux at a power level of about $2.45 \mathrm{~W}$. Then, in either case, the temperature everywhere increases sharply as the entire flow is in single vapor phase with the thermal conductivity of the working fluid dropping sharply.

It is important to keep in mind that for proper boiling curves, either the fluid or wall temperature is required as a function of the heat removed by the working fluid. It is still difficult to integrate microsensors within the bulk fluid flow or on the inner surface of a microchannel to provide such temperature measurements. Therefore, only the device surface temperature measurements have been reported thus far. Moreover, a portion of the input power is stored as internal energy of the entire chip (and package), which may not be negligible. Still further, in cases of high device temperature and small flow rate, natural heat convection from the device surface to the ambient may not be negligible compared with the forced heat convection by the working fluid flow.

\section{Two-phase flow patterns}

Two-phase flow patterns in ducts are the result of the detailed heat transfer between the solid boundary and the working fluid. The flow patterns are important since the temperature distributions in both the solid boundary and the fluid flow are directly determined by these patterns. Various hydrodynamic conditions are encountered when a duct is heated and fed with subcooled liquid at its entrance at such a rate that the liquid is totally vaporized at the duct exit. Although gravity is important in macro-scale convective boiling, it has no effect on the flow pattern evolution in a microchannel during boiling described hereafter.

\subsection{Bubble formation and dynamics}

Bubble activity was reported in triangular microchannels with a hydraulic diameter of $26 \mu \mathrm{m}$ [5]; there, it was speculated that bubbly flow pattern had not been observed due to size effect. The current experiments re-confirm that as long as a nucleation site is active, a periodic cycle of bubble formation, growth and departure can be observed inside a $14 \mu \mathrm{m}$ deep, nearly rectangular microchannel as documented in the picture series of figure 8 . The bubble activity started at input power level as low as $q / q_{\mathrm{CHF}} \cong 0.65$ when the water was forced through the microsystem under inlet pressure of $45 \mathrm{kPa}$, resulting in an average device temperature of about $70{ }^{\circ} \mathrm{C}$. Regardless of whether the origin of the bubble was dissolved gas or liquid phase change, bubbles could be seen forming at specific locations along the channel walls at a few nucleation sites with constant frequency. The channel interior surfaces were very smooth due to the micro fabrication processes. Therefore, very few active nucleation sites were observed; almost always at defects along the channel top surface, e.g. pinholes, as demonstrated in figure $8(a)$. Once formed, the bubble then grew in the flow direction until it filled the entire channel crosssection as seen in figure $8(b)$. Since the bubble could not grow any further due to the geometric constraint, figure $8(c)$, it was stretched away in the flow direction while still anchored to the nucleation site. Once a critical force balance was developed, the bubble departed from the nucleation site triggering a new cycle as shown in figure $8(d)$.

An interesting feature, to which the small channel size probably contributed, was the bubble evolution following its departure. In the classical process, the vapor bubbles recondense in an abrupt process (collapse), thus, transferring heat to colder regions in the flow field and maintaining smaller gradients in the temperature field of the working 


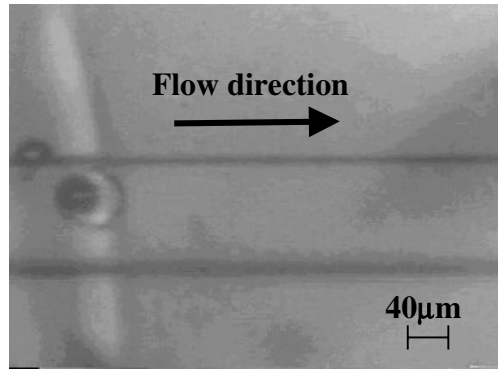

(a)

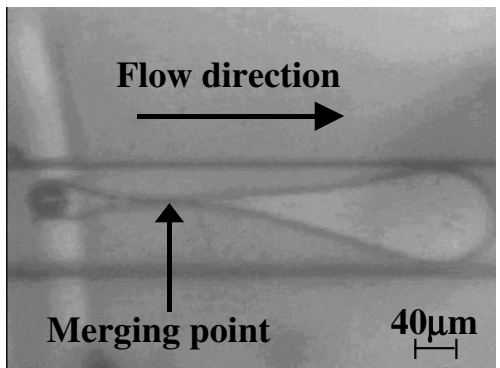

$(c)$

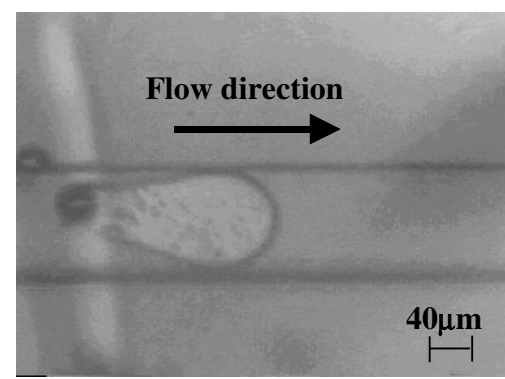

(b)

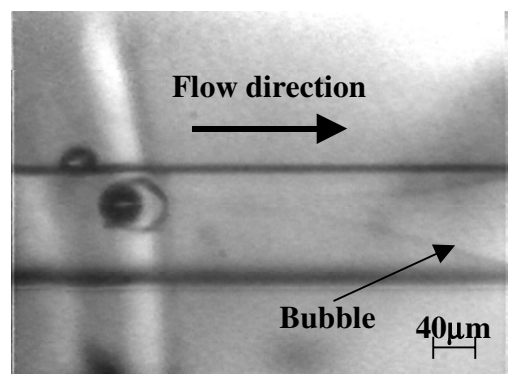

(d)

Figure 8. Bubble activity in an active nucleation site demonstrating (a) bubble formation, $(b)$ bubble growth, $(c)$ bubble on the verge of departure and $(d)$ bubble after departure.

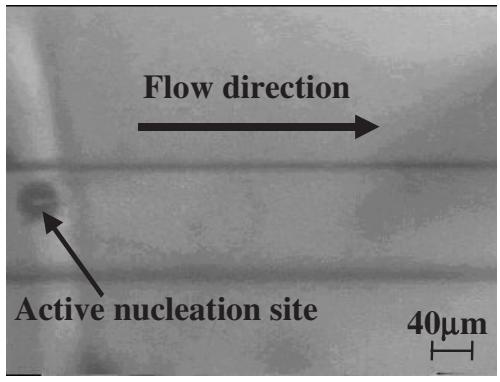

(a)

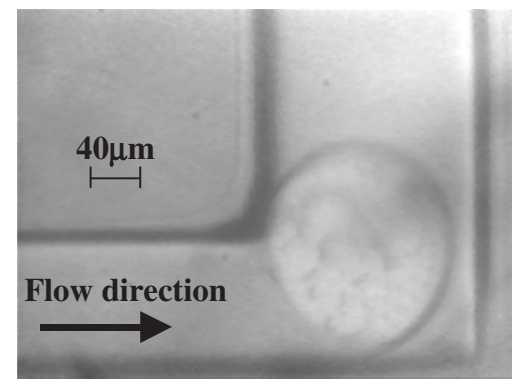

$(b)$

Figure 9. Bubble motion through the microchannel: $(a)$ bubble disappearance following its departure from the nucleation site and (b) bubble re-formation upon impact on the channel exit wall.

fluid. However, in the present case, the bubbles did not recondense but, as viewed by a regular-speed camera in the pictures shown in figure 9, seemed to travel undetected at a very high speed along the channel. The bubbles disappeared immediately following their departure from the nucleation site, figure $9(a)$. The bubbles then re-formed, after a certain time delay, upon impingement on the channel exit wall, figure $9(b)$. Subsequently, the bubbles continued to move toward the device single outlet and out of the system. The bubble motion seemed to be adiabatic with no heat transfer to the surrounding liquid, since the re-formed bubble size was about the size of the departed one. These observations suggest that the bubbles had originated from dissolved gas in the water since the device temperature, about $70{ }^{\circ} \mathrm{C}$, was far below the water saturation temperature. Consequently, the departing bubbles did not condense back to liquid phase, but rather re-formed upon impact on the microchannel exit wall.

The physical mechanism responsible for bubble departure in a micro domain may be different from classical models. Figure $8(c)$ suggests that the bubble departed when the two vapor/liquid interfaces merged together along the stem connecting the bubble to the nucleation site. Consequently, the bubble was snapped off from the nucleation site, and was free to move within the fluid flow. This should be considered a direct result of the small channel size, since the bubble growth is limited by the channel solid walls and not by surface tension or vapor condensation. Flow visualizations using a high-speed camera revealed a sequence of flow patterns that had not been detected by a regular camera. In subcooled nucleate boiling, vapor bubbles grow to a critical size prior to their departure and condense in the cold liquid. However, due to the limited space in a microchannel, the bubble grows until it occupies the entire cross-section. Resting against the solid walls, the bubble is then stretched in the flow direction. A very long stem, bounded by two vapor-liquid interfaces, connects the bubble to its nucleation site as shown in figure 10(a). Only when the surface tension along the stem is strong enough to pull the interfaces inward overcoming the vapor pressure in the stem such that both interfaces merge does the bubble depart. The bubble then loses its distinct boundary, as indicated in figure $10(b)$, but does not condense; instead the bubble re-forms upon impact 


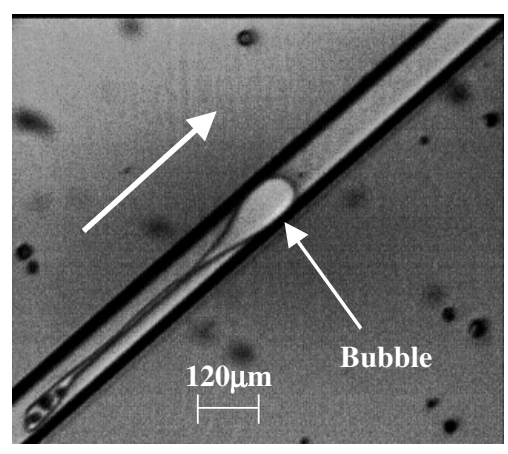

(a)

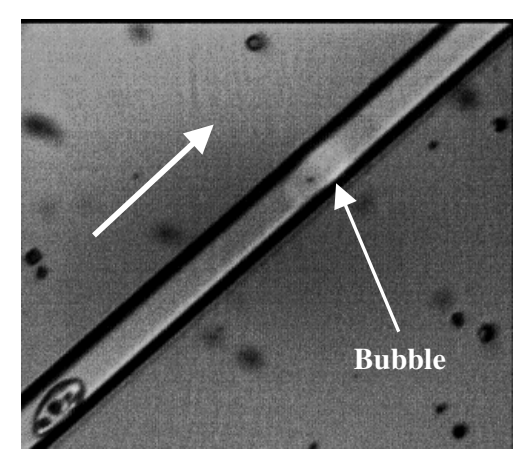

(b)

Figure 10. High-speed camera pictures of (a) mature bubble with a stretched stem and $(b)$ departed bubble following the merger of both interfaces bordering the vapor stem.

M An MPEG movie of this figure is available from stacks.iop.org/JMM/13/155

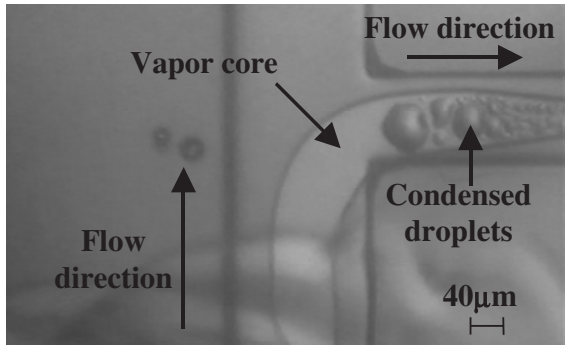

(a)

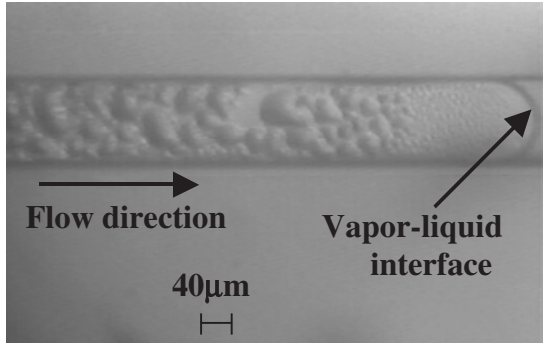

(b)

Figure 11. Pictures of two-phase flow pattern: $(a)$ around the channel entrance at $q / q_{\mathrm{CHF}} \sim 0.9$ and $(b)$ next to the channel exit at $q / q_{\mathrm{CHF}} \rightarrow$ $1\left(Q_{\mathrm{L}}=0.0315 \mathrm{~mL} \mathrm{~min}^{-1}\right)$.

at the channel exit indicating that it originated from dissolved gas.

The local nucleation mode was observed for both flow rates over an input power range of $0.65<q / q_{\mathrm{CHF}}<0.85$. The effect of this mode on the overall heat transfer is negligible, since no clear change can be seen in the trend of the boiling curves (figure 7). Increasing the input power level to $q / q_{\mathrm{CHF}} \sim$ 0.9 resulted in the development of unstable flow patterns, and bubble nucleation inside the microchannel could no longer be detected. Clearly, the suppression of the nucleation sites, not observed in macrochannels, was the result of the evolution of a more dominant two-phase flow mode rather than a genuine size effect. It is reasonable to expect that the bubble dynamics after inception, unlike the nucleation site density, is affected by the channel size. Still it has been demonstrated that in microchannels, with channel height as small as $14 \mu \mathrm{m}$, bubble growth and departure are possible. Thus, the lack of partial nucleate boiling of subcooled liquid flowing through microchannels cannot be attributed to a direct fundamental size effect suppressing bubble dynamics, i.e. bubbles cannot grow and depart due the small size of the channel. However, it is very plausible that the absence of partial nucleate boiling is an indirect size effect. Namely, other boiling modes become dominant due to small channel size, i.e. strong capillary forces, and as a result bubble nucleation is suppressed.

\subsection{Transient flow patterns}

In triangular microchannels, a stable annular flow superseded the local nucleation with increasing input power [5]. It is not clear whether the stable annular flow is a general pattern in microchannels, due to size effect, or it is unique only to the triangular channel cross-section due to the strong capillary forces at the sharp corners (similar to micro heat pipes). Therefore, in the current device, the channel cross-section is modified from triangular to nearly rectangular, figure 3 , to test the possible channel shape effect on the evolving flow patterns. At a low water flow rate, the average flow velocity in the microchannels was about $34 \mathrm{~mm} \mathrm{~s}^{-1}$. This low speed provides sufficient time for the fluid to exchange heat with the ambient through the 'hot' silicon and 'cold' glass substrates, resulting in vapor condensation on the glass inner surface. Furthermore, the large aspect ratio of the channel rectangular cross-section, about 8.5 , resulted in a large surface area for free convection heat transfer. Thus, the combination of low flow velocity and large free convection area allowed a significant portion of the heat to be conducted through the device and transferred to the surrounding. The loss of heat was accompanied by recondensation of the vapor phase forming liquid droplets on the colder glass ceiling, as shown in figure 11(a), even at the channel inlet next to the heat source. Since the heater was located near the inlet, the channel wall temperature decreased towards the heat sink outlet. Consequently, a transition region between the upstream vapor and the downstream liquid was established as shown in figure $11(b)$. Close to the onset of CHF condition, the transition region shifted downstream toward the channel exit. The flow void fraction increased with increasing power not only due to the streamwise motion of the transition region but also due to re-vaporization of the liquid droplets. At higher water velocity with smaller free convection area, 


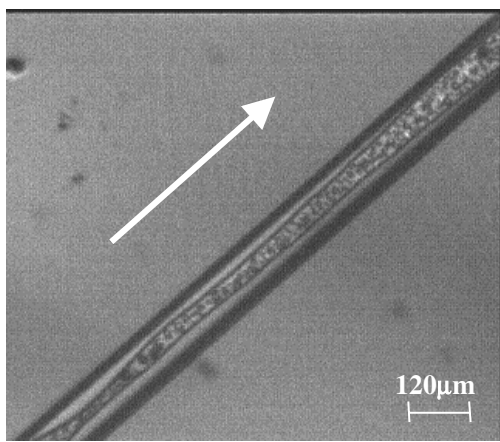

(a)

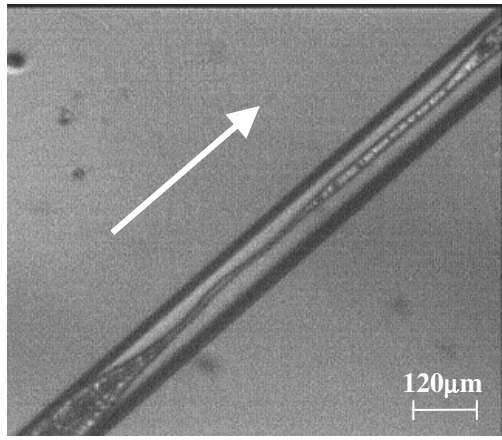

$(c)$

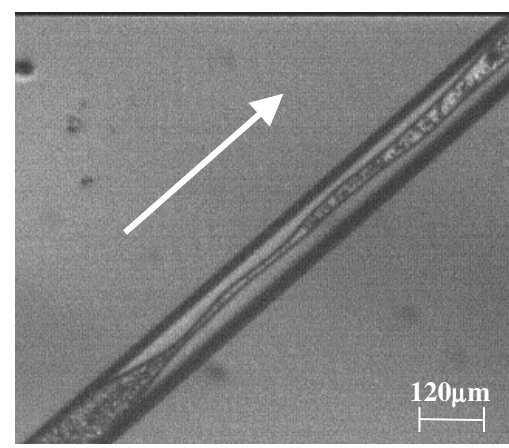

(b)

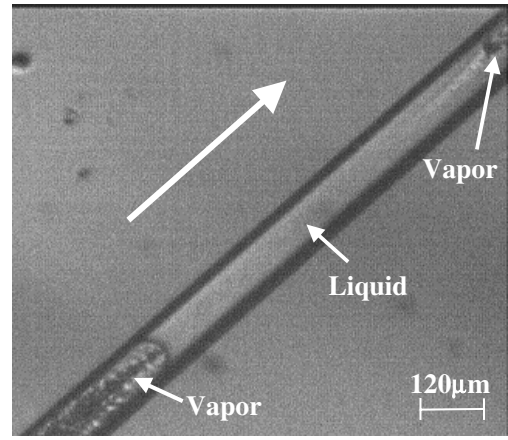

(d)

Figure 12. High-speed camera picture series, $(a)-(d)$, of formation and collapse of an unstable annular flow in a nearly rectangular microchannel about $14 \mu \mathrm{m}$ in depth and $120 \mu \mathrm{m}$ in width.

M] An MPEG movie of this figure is available from stacks.iop.org/JMM/13/155

vapor re-condensation did not occur as no liquid droplets were observed in the vapor core.

No annular flow was observed in the rectangular channel when visualizing the convective boiling under a regular CCD camera. However, using a high-speed camera, 500 frames per second, an annular flow field was detected in the microchannel as demonstrated in the picture series in figure 12. This flow mode was highly unstable and, within $0.3 \mathrm{~s}$ of its formation (figure 12(a)), the liquid films on each side of the vapor core were attracted to each other due to surface tension (figure 12(b)). After the merger of the two liquid films (figure 12(c)), the vapor core snapped immediately and the annular flow mode disappeared (figure 12(d)). This is drastically different from the triangular microchannels, where the annular flow was the most stable mode over a large range of input power, practically up to the onset of critical heat flux.

\subsection{Dominant transition flow-pattern mode}

At the high input power range, $q / q_{\mathrm{CHF}}>0.9$, the most prevalent boiling flow pattern observed in a rectangular microchannel was a transition region separating an upstream vapor zone from a downstream intermittent zone. The transition region seemed to oscillate randomly along the microchannel with no clear structure. However, analyzing the individual still pictures from the CCD camera, a fascinating orderly pattern was observed as detailed in figure 13. Upstream of the transition region (figure 13(a)), the flow was in single vapor phase since the heater was located at the channel inlet. At the upstream tail of the transition region (figure 13(b)), liquid droplets started to condense on the colder channel glass ceiling. The liquid droplets increased in number and size (figure 13(c)) until two liquid films were condensed on both sidewalls (figure 13(d)). Thus, an annular flow pattern was formed over a short segment (figure 13(e)). The liquid films became wider until they touched each other (figure 13(f)). When this occurred, a vapor section at the head of the transition region was severed from the upstream vapor zone (figure $13(g)$ ). Finally, after the two liquid films merge together, the upstream vapor region was completely snapped off (figure 13(h)), leaving a vapor segment bounded by liquid flow. The entire transition region retreated far upstream, and the cycle repeated itself resulting in an intermittent flow downstream of the transition region.

The transition region oscillated violently along the microchannel with rather large amplitude, and its average location depended on the input power. For the low flow rate of $0.0315 \mathrm{~mL} \mathrm{~min}{ }^{-1}$, the transition region length from its tail (figure $13(b)$ ) to its front at the vapor-liquid interface (figure $13(g)$ ) was about $2-3 \mathrm{~mm}$. Initially, at a power level corresponding to the start of the boiling plateau (figure $7(a)$ ), $q / q_{\mathrm{CHF}} \sim 0.9$, the transition region developed near the channel inlet. As the input power increased, the transition region moves downstream appearing around the channel outlet when the input power reached the CHF level. Thus, the additional power is used to vaporize more liquid, and the vapor content increased as the transition region moved downstream with no temperature increase. When the transition region was at the exit, all the working fluid was in vapor phase giving rise 


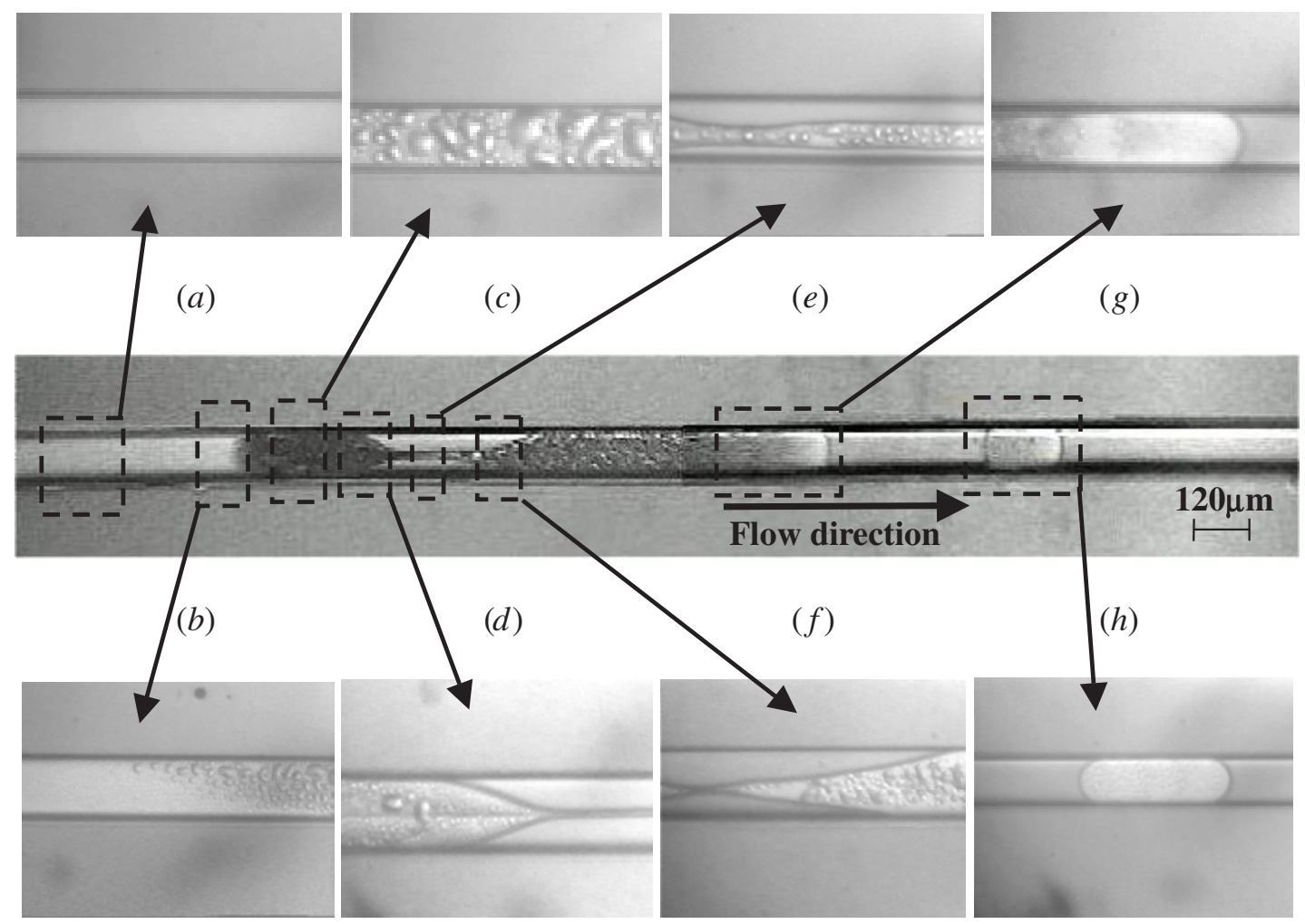

Figure 13. A sequence of pictures, $(a)-(h)$, detailing the transition region from the upstream vapor $(a)$ to the downstream liquid zone $(h)$ in two-phase convective boiling flow. Flow direction is from left to right $\left(Q_{\mathrm{L}}=0.0315 \mathrm{~mL} \mathrm{~min}^{-1}\right)$.

M. An MPEG movie of this figure is available from stacks.iop.org/JMM/13/155

to the onset of $\mathrm{CHF}$, which was accompanied by the sharp increase of the temperature. However, for the high flow rate of $0.063 \mathrm{~mL} \mathrm{~min} \mathrm{~m}^{-1}$, the length of the transition region was about $11 \mathrm{~mm}$. Since the total length of the microchannels was $19 \mathrm{~mm}$, the transition region with its oscillations practically occupied the entire channel. Consequently, increasing the input power resulted in increasing temperature with no boiling plateau (figure $7(b)$ ). The CHF condition was easily triggered once the unstable annual flow suddenly developed in the microchannals.

\section{Physical modeling}

Apparently the channel cross-section geometry does have an effect on the evolving flow pattern due to convective flow boiling in microchannels. In the triangular microchannels, a stable annular flow was established at a relatively low input power suppressing the development of bubbly flow [5]. In contrast, in the rectangular microchannels, the annular flow was highly unstable giving rise to an oscillating transition region as the dominant flow pattern.

Annular flow mode typically includes a continuous thin liquid film coating the inner channel surface with no contact lines as illustrated in figure 14(a). However, the sharp interfaces reported for the triangular channel ceiling [5] suggest the existence of contact lines as sketched in figure 14(b). This flow pattern indeed appears to be very stable in the triangular cross-section. Physically, hydrodynamic stability requires an intrinsic restoring force to damp out naturally occurring flow perturbations. Without such a

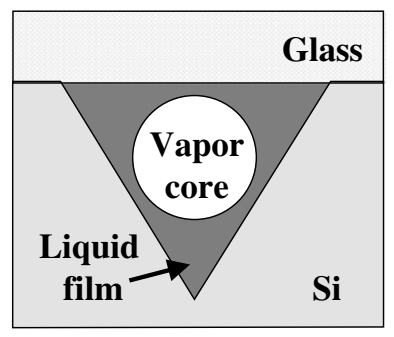

(a)

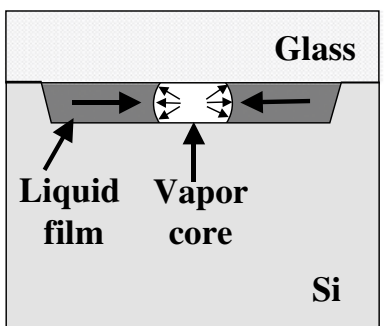

(c)

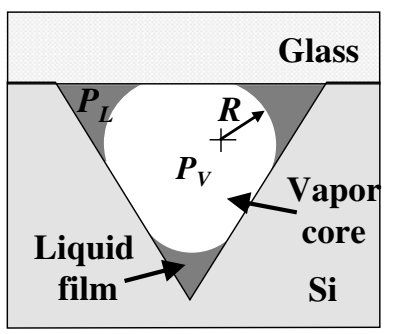

(b)

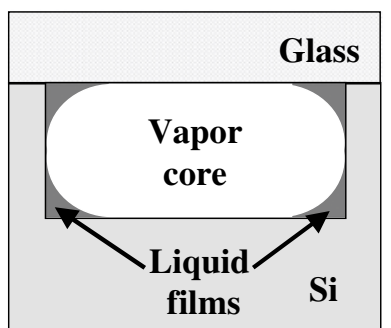

(d)
Figure 14. Schematic illustrations of an annular flow mode in $(a)$ a triangular cross-section with a continuous liquid film, $(b)$ a triangular cross-section with contact lines, $(c)$ a shallow rectangular cross-section with contact lines across the channel and $(d)$ a deep rectangular cross-section with contact lines at the corners.

restoring force, the perturbations can either stay at a constant level, marginally stable, or grow leading to flow instability. In the current system, the balance of forces across the 
vapor-liquid interface is given by the Young-Laplace equation

$$
P_{\mathrm{V}}-P_{\mathrm{L}}=\Delta P=\frac{\sigma}{R}
$$

where $P_{\mathrm{V}}$ and $P_{\mathrm{L}}$ are the vapor and liquid pressure, while $\sigma$ and $R$ are the liquid surface tension coefficient and the interface radius of curvature, respectively. If the vapor pressure decreases or the liquid pressure increases, $\Delta P \downarrow$, the interfaces move inward to the channel center resulting in a larger radius of curvature, $R \uparrow$ increases, and smaller surface tension force, $(\sigma / R) \downarrow$ decreases. If the vapor pressure increases or the liquid pressure decreases, $\Delta P \uparrow$, the interfaces move outward deeper into the corners leading to a smaller radius of curvature, $R \downarrow$, and higher surface tension force along the interfaces, $(\sigma / R) \uparrow$. Thus, due to the triangular configuration, a restoring force exists, to damp out pressure perturbations along the annular flow rendering it highly stable.

In contrast, this restoring force is absent in the nearly rectangular microchannels. The interfaces seem to span the entire channel height, as shown in figure 14(c). Spanwise interface motion due to pressure perturbations, either $\Delta P \downarrow$ or $\Delta P \uparrow$, does not lead to changes in the radius of curvature and the surface tension force stays constant, $(\sigma / R)=$ const. In a rectangular microchannel, therefore, the liquid film/vapor core interface fluctuates due to pressure perturbations without a restoring force. Consequently, if the vapor pressure drops, the two interfaces on either side of the vapor core move inward without additional resistance. This movement can result in the merger of the two liquid films at some point along the channel, as demonstrated in figure $12(c)$, rendering the annular flow highly unstable. Of course, this instability is the result of not only shape but also size effect. It is straightforward to imagine a rectangular microchannel with a larger height as drawn in figure $14(d)$. In such a case, known to develop in rectangular heat pipes, the liquid/vapor interface does not extend across the entire channel height; instead, the liquid is confined in each corner by an interface similar to the triangular configuration in figure 14(b). Following the same restoring force argument, the annular flow in a deep rectangular channel should also be stable and probably the dominant flow mode in convective boiling. It is interesting to note that the mechanism identified as responsible for the instability of annular flow in a shallow rectangular microchannel is also the same physical mechanism responsible for: (i) the bubble departure demonstrated in figure 10 and (ii) the establishment of intermittent flow downstream of the transition region shown in figure 13.

\section{Conclusions}

A nearly rectangular microchannel heat sink integrated with a local heater and array of temperature microsensors has been fabricated and characterized. Water was forced through the microchannels, about $14 \mu \mathrm{m}$ in depth, under a constant inlet pressure. The device surface temperature was measured as a function of input power to establish the boiling curves. The typical plateau in the boiling curves was observed at a lower flow rate, where a 'short' transition region developed. Increasing the input power resulted in downstream movement of the transition region increasing the void fraction. Thus, the increased power was essentially converted into latent heat without increasing the device temperature. However, at the higher flow rate, the transition region occupied almost the entire channel. Increasing the input power resulted in increased device temperature and no plateau was observed in the boiling curve.

Similar to previous reports, local nucleation inside the microchannels was observed. As long as an active site existed, typically a pinhole in the channel upper surface, bubbles could form, grow and depart. Partial nucleate boiling, or bubbly flow, was not observed due to the development of more dominant, size and shape dependent flow modes suppressing local nucleation in the microchannels. Unlike in triangular microchannels, where a stable annular flow was the dominant flow pattern, a transition region separating an upstream vapor from a downstream liquid zone was found to be the dominant flow pattern in the rectangular microchannels. Although appeared to be disorderly, the transition region contained a definite sequence of two-phase flow patterns, from droplets in vapor flow at its tail to a clear vapor-liquid interface at its front.

A physical mechanism has been proposed to explain the difference between the flow patterns observed in triangular and rectangular microchannels. Based on the force balance across a vapor-liquid interface, annular flow in triangular microchannels is stable due to the development of a size and shape dependent restoring force damping out naturally occurring perturbations. Similar restoring force cannot develop in shallow rectangular microchannels allowing the merger of two liquid films on either side of a vapor core. The merging of the liquid films and disconnecting the vapor core are identified as the mechanisms responsible for the: (i) bubble departure, (ii) instability of annular flow and (iii) development of intermittent flow downstream of the transition region in shallow rectangular microchannels.

\section{Acknowledgment}

This work is supported by the Hong Kong Research Grant Council through grant HKUST6212/01E.

\section{References}

[1] Stanley R S, Ameel T A and Warrington R O 1995 Convective flow boiling in microgeometries: A review and applications Proc. Convective Flow Boiling Conf. (Canada) pp 305-10

[2] Jiang L, Wong M and Zohar Y 2000 Transient temperature performance of an integrated micro-thermal system J. Micromech. Microeng. 10 466-76

[3] Hetsroni G, Mosyak A and Segal Z 2001 Nonuniform temperature distribution in electronic devices cooled by flow in parallel microchannels IEEE Trans. Compon. Packag. Technol. 24 16-23

[4] Peng X F and Wang B-X 1993 Forced convection and flow boiling heat transfer for liquid flowing through microchannels Int. J. Heat Mass Transfer 36 3421-7

[5] Jiang L, Wong M and Zohar Y 2001 Forced convection boiling in a microchannel heat sink J. Microelectromech. Syst. 10 80-7

[6] Peles Y P, Yarin L P and Hetsroni G 2001 Steady and unsteady flow in a heated capillary Int. J. Multiph. Flow 27 277-98

[7] Zhang L, Koo J M, Jiang L, Goodson K E, Santiago J G and Kenny T W 2001 Study of boiling regimes and transient signal measurements in microchannels 11th Int. Conf. Solid-State Sensors and Actuators pp 1514-7 
[8] Zhang L, Wang E N, Koo J M, Jiang L, Goodson K E, Santiago J G and Kenny T W 2002 Enhanced nucleate boiling in microchannels Proc. 15th Micro Electro Mechanical Systems Conf. (USA) pp 89-92

[9] Lee M, Wong Y Y, Wong M and Zohar Y 2001 Two-phase flow boiling in microchannel heat sink pro. ASME Int.
Mechanical Engineering Congress and Exposition (New York)

[10] Jiang L, Wong M and Zohar Y 1999 Phase change in microchannel heat sinks with integrated temperature sensors J. Microelectromech. Syst. 8 $358-65$ 